\title{
Tomographic Wave-Front Sensing with a Single Guide Star
}

\author{
Michael Hart ${ }^{* a, c}$, Stuart Jefferies ${ }^{\mathrm{b}, \mathrm{c}}$, and Douglas Hope ${ }^{\mathrm{d}}$ \\ ${ }^{a}$ College of Optical Sciences, University of Arizona, 1630 E. University Blvd., Tucson, AZ 85721 \\ bepartment of Physics and Astronomy, Georgia State University, Atlanta, GA 30303 \\ 'Institute for Astronomy, University of Hawaii, 34 Ohia Ku Street, Pukalani, HI 96768 \\ ${ }^{\mathrm{d}}$ Center for Space Situational Awareness Research, US Air Force Academy, Colorado Springs, CO 80848
}

\begin{abstract}
Adaptive optics or numerical restoration algorithms that restore high resolution imaging through atmospheric turbulence are subject to isoplanatic wave-front errors. Mitigating those errors requires that the wave-front aberrations be estimated within the 3D volume of the atmosphere. Present techniques rely on multiple beacons, either natural stars or laser guide stars, to probe the atmospheric aberration along different lines of sight, followed by tomographic projection of the measurements onto layers at defined ranges. In this paper we show that a three-dimensional estimate of the wave-front aberration can be recovered from measurements by a single guide star in the case where the aberration is stratified, provided that the telescope tracks across the sky with non-uniform angular velocity. This is generally the case for observations of artificial earth-orbiting satellites, and the new method is likely to find application in ground-based telescopes used for space situational awareness.
\end{abstract}

Keywords: wave-front sensing, tomography, anisoplanatism, telescopes

\section{INTRODUCTION}

The three-dimensional nature of the atmosphere imposes anisoplanatic effects on ground-based telescopes designed to deliver high-resolution imaging. Turbulence broadly distributed along the line of sight introduces angular anisoplanatism, a strong field dependence in the point-spread function. Adaptive optics (AO) systems that rely on a laser guide star (LGS) are additionally subject to focal anisoplanatism arising because the volume of air sampled by the beacon at finite range differs from that encountered by a more distant object. To achieve blur-free imagery in either case, the wave front must first be characterized over the volume of atmosphere between the scene and the telescope.

In nighttime astronomy this is achieved by wave-front sensor (WFS) measurements of a constellation of guide beacons, lasers or natural stars, that surround the target of interest. The same approach is used in AO for solar astronomy, except here a number of small regions of the solar surface that contain significant high spatial frequency information at appreciable contrast, replace the guide stars as beacons. ${ }^{1,2}$ Each region is projected onto a Shack-Hartmann WFS that forms a set of images as observed through the sensor's sub-apertures. The images are each cross-correlated with a reference image and the local wave-front phase gradients obtained from the positions of the peak values.

As presently practiced, tomographic wave-front sensing can be thought of as a two-step process..$^{3-5}$ To begin, line integrals of a quantity closely related to the atmospheric aberration such as its first spatial derivative, are measured by the WFS along a number of discrete lines of sight. A tomographic algorithm then remaps the integrands onto horizontal layers defined at discrete altitudes in the atmosphere. The mapping is typically constrained to minimize the mean-square difference between the measured quantities and the corresponding line integrals projected through the atmospheric model constructed from the tomographic analysis. ${ }^{6}$

Our approach departs from this paradigm. We show in this paper that tomographic wave-front information can also be recovered from a single beacon provided that its tracking rate is not constant. The variation in the track rate overcomes a degeneracy in the WFS measurements that otherwise does not allow range information to the aberration to be inferred. Non-constant track rates are generally not encountered in astronomical observations, but frequently will be when the objects are orbiting man-made satellites. For that reason, we believe the method will be valuable for high-resolution observations of satellites made from the ground.

*mhart@optics.arizona.edu, +1-520-626-5265

Unconventional Imaging and Wavefront Sensing XII, edited by Jean J. Dolne, Thomas J. Karr, David C. Dayton, Proc. of SPIE Vol. 9982, 998207 · @ 2016 SPIE · CCC code: 0277-786X/16/\$18 · doi: 10.1117/12.2257358 


\section{EXPLOITING FROZEN FLOW BEHAVIOR}

Our method exploits the fact that the aberration is strongly correlated on short time scales. We use the frozen flow model (FFM) which treats the evolution of the aberration as a series of independent static layers, each moving across the telescope aperture with the prevailing wind at the altitude of the layer. Because of its simplicity, the FFM is frequently used as the basis for numerical studies of telescope imaging performance, particularly in the modeling of AO systems. While the FFM is observed not to hold in the real world over long time scales, a number of studies ${ }^{7-9}$ have shown that it is a reasonable approximation for short but still interesting periods. For example, from observations made at the $1.5 \mathrm{~m}$ telescope of the Starfire Optical Range in New Mexico, at $0.74 \mu \mathrm{m}$ wavelength, Schöck \& Spillar ${ }^{9}$ found that the FFM is a good approximation for a time scale $\tau_{\mathrm{FFM}}$ of $20 \mathrm{~ms}$ or less. The accuracy degraded over time such that after $100 \mathrm{~ms}$ only $50 \%$ of the temporal evolution of the wave front could be described by the FFM. Nevertheless, the period of validity of the model, $\tau_{\mathrm{FFM}}$, is an order of magnitude longer than $\tau_{0}$, the expectation value of the time required for the phase of a wave front in a circular telescope aperture to change by 1 radian rms. ${ }^{10}$

We take advantage of the FFM in three ways. Firstly, a spatio-temporal autocorrelation of the WFS data reveals the number of layers of significant aberration in the atmosphere. Secondly, the shear that develops over successive WFS frames between these layers allows their detailed contributions to the aberration in the pupil to be separated. Finally, as described below, the autocorrelation provides measurements of the angular velocity of each layer through the WFS beam from which the ranges may be estimated.

In the very simple and artificial case of aberration characterized by a single frozen but wind-blown layer, it is easy to see that taking WFS data at two different times along the same line of sight can yield tomographic information in the same way as two simultaneous measurements along different lines of sight. The equivalent angular separation of the sight lines $\theta_{\mathrm{s}}$ in the former case is $v_{1} t_{\mathrm{WFS}} / h_{1}$ where the quantities are respectively the wind speed $v_{1}$ at the layer, the elapsed time $t_{\mathrm{WFS}}$ between frames of the WFS, and the range of the layer $h_{1}$ from the telescope. In standard multi-beacon tomography, $\theta_{\mathrm{s}}$ is determined by the geometry of the optical system and is known. However, in the single-beacon case, only $t_{\mathrm{WFS}}$ is known. To recover the same wave-front information one must estimate both $v_{1}$ and $h_{1}$.

Because we believe our method will be of most value in imaging artificial satellites, which often requires the use of a LGS, our analysis in the remainder of the paper explores the case of a beacon at finite range. The result, however, is equally applicable to guide stars at any range. The geometry and nomenclature are established in Figure 1.

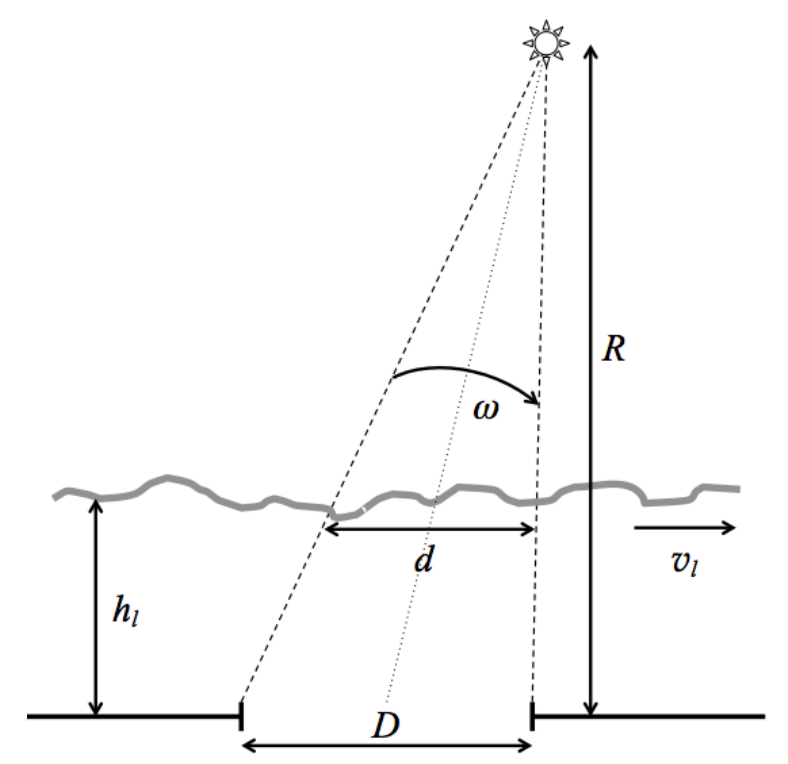

Figure 1. Wave-front sensing geometry analyzed in this paper. A LGS at range $R$ illuminates a telescope of diameter $D$. A layer of atmospheric aberration at height $h_{l}$ propagates with speed $v_{l}$. The LGS beam footprint at the layer has diameter $d$. The telescope tracks with angular speed $\omega$. 


\section{LAYER DEFINITION}

We begin with an analysis of the WFS data to determine the number of layers of significant atmospheric aberration and their apparent velocity through the telescope's light path. The technique, described in detail by Hope et al., ${ }^{11}$ relies on the 3D spatio-temporal autocorrelation of the wave-front slopes calculated from the signals of a Shack-Hartmann WFS. The signature of a frozen layer is a line of high correlation radiating from the origin. The angles made by the line with respect to the zero-spatial-lag axis in the autocorrelation cube are determined by the apparent wind vector of the layer. The coherence time $\tau_{\mathrm{FFM}}$ for the layer is estimated from the rate of decay of the correlation along the line, and although we do not use this information, the power in the line indicates the strength of the aberration. Figure 2 shows a number of consecutive time-lag slices from an autocorrelation cube calculated from WFS data recorded at the AEOS $3.6 \mathrm{~m}$ telescope in Hawaii. Peaks can be seen that correspond to three distinct layers above the telescope, as well as other peaks which do not lie on lines coming from the origin and reflect random correlations.
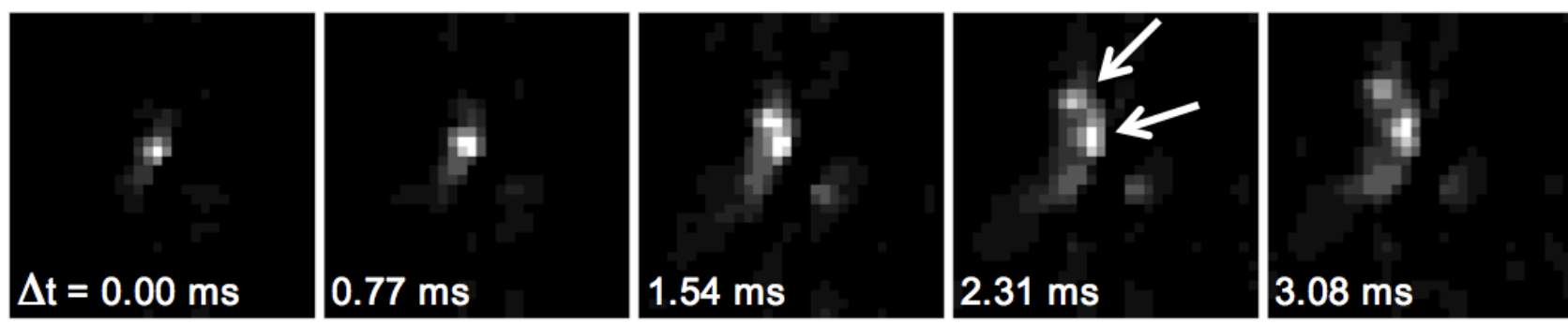

Figure 2. Consecutive time-lag slices from the 3D autocorrelation of Shack-Hartmann WFS data. The three white arrows indicate correlation peaks corresponding to frozen aberration layers propagating with different velocities. The shaded arrow indicates a random peak not associated with a frozen layer.

In the next step, ${ }^{11}$ we employ a non-linear least-squares technique in a partial separation of the wave-front gradients on the layers we have identified. The estimates are constrained to match the WFS data in both space and time. Phases at each layer are then calculated from the gradients in the usual way. (Although not germane to tomography, the spatial subsampling afforded by the motion of each layer allows us to recover phase estimates on spatial scales shorter than the Nyquist limit imposed by the size of the WFS sub-apertures. ${ }^{12}$ )

\section{NUMERICAL SIMULATION}

We have built a small simulation to show this layer separation. Four atmospheric layers having different strengths, wind vectors, and heights were modeled with frozen Kolmogorov turbulence, as illustrated in Figure 3. A sequence of 1000 phase screens in the pupil of a telescope of $4 \mathrm{~m}$ diameter was made by propagating the layers in time, and light from a beacon through the layers. The beacon was taken to be at $90 \mathrm{~km}$ range, the approximate height of a sodium LGS. The WFS was modeled as a Shack-Hartmann with a grid of $32 \times 32$ sub-apertures running at 1300 frames per second. The parameters of the model atmosphere are listed in Table 1. The effective value of the Fried coherence length $r_{0}$ at $500 \mathrm{~nm}$ wavelength was $9.7 \mathrm{~cm}$, and the coherence time $\tau_{0}$ was $2.0 \mathrm{~ms}$. The mean height of the aberration was $5400 \mathrm{~m}$, giving an isoplanatic angle of 1.1 arc seconds.

Table 1. Parameters of the model atmosphere.

\begin{tabular}{ccccc}
\hline Layer & $\boldsymbol{r}_{\mathbf{0}}(\mathbf{c m})^{*}$ & Height $(\mathbf{m})$ & Direction & Speed $(\mathbf{m} / \mathbf{s})$ \\
\hline 1 & 17 & 0 & $0^{\circ}$ & 2 \\
2 & 20 & 500 & $0^{\circ}$ & 10 \\
3 & 30 & 5000 & $-10^{\circ}$ & 25 \\
4 & 30 & 15000 & $40^{\circ}$ & 50 \\
\hline \multicolumn{4}{c}{ *Value of the Fried parameter at 500 nm wavelength. }
\end{tabular}



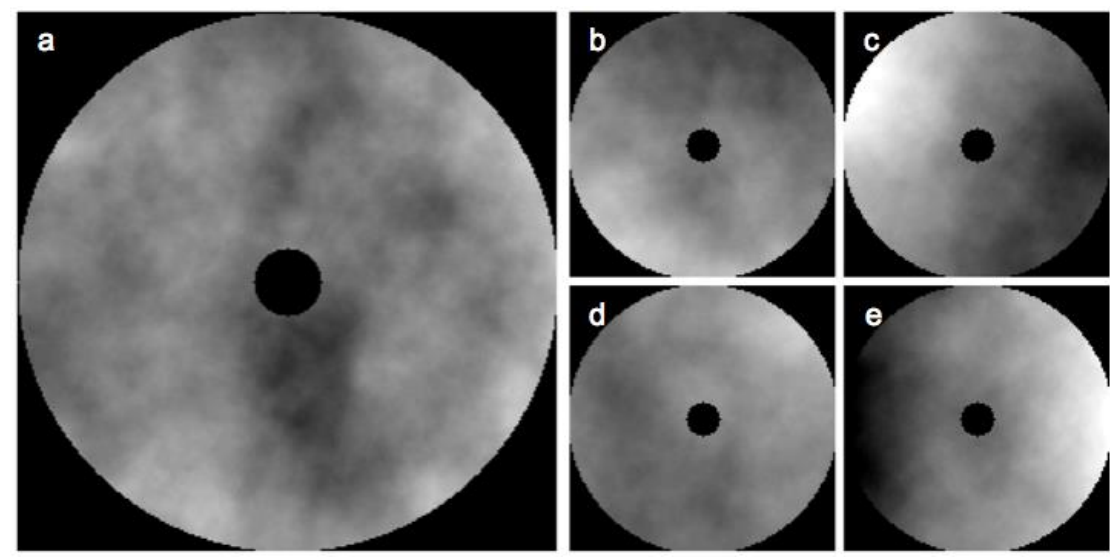

Figure 3. Single frame from the atmospheric simulation. Combined aberration $a$ is the sum of the four layers $b$ through $e$.

The effect of the layer separation is shown in Figure 4 which compares the angular anisoplanatism as a function of field angle for two cases. In the first, all the aberration is taken to be in the telescope pupil, the conventional assumption for AO systems. In the second, the upper layer was separately estimated and taken to be at the correct range of $15 \mathrm{~km}$. The lower three layers were estimated as a single layer, placed in the pupil.

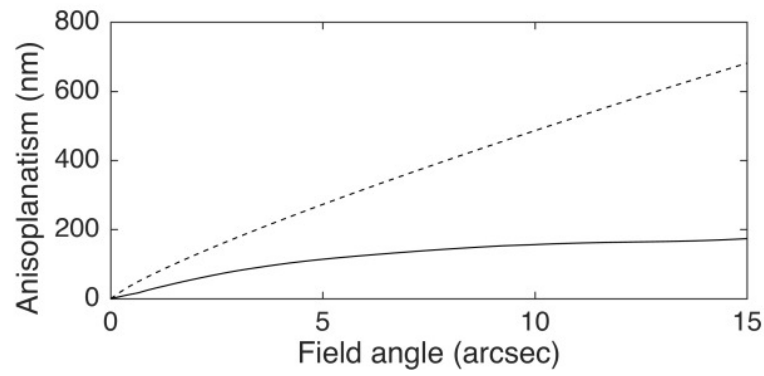

Figure 4. Angular anisoplanatism for conventional wave-front compensation in the pupil plane only (dashed line) and for two altitude-conjugated correcting layers (solid line) applied to the modeled four-layer atmosphere.

At this point, although the short-term frozen flow character of the atmosphere has allowed us to determine the number of significant layers of aberration and to distinguish the detailed moment-by-moment contributions, we have still not established the layer ranges, and so cannot make use of our knowledge to mitigate either angular or focal anisoplanatism. The ranges are not uniquely constrained by the data. We can break the degeneracy however if observations can be made with the telescope tracking at two different angular velocities.

Referring to Figure 1, we start by writing the time $t_{c}$ for a point on a single layer to cross the telescope beam,

$$
t_{c}=d /\left|\vec{v}_{r}\right|
$$

where superscript arrows represent vector quantities. The beam footprint diameter $d=D\left(1-h_{l} / R\right)$, and the resultant velocity of the beam through the layer is given by

$$
\vec{v}_{r}=\vec{v}_{l}-\vec{\omega} h_{l} .
$$

Hence,

$$
t_{c}=\frac{D\left(1-h_{l} / R\right)}{\left(v_{l}^{2}+\omega^{2} h_{l}^{2}-2 v_{l} \omega h_{l} \cos A\right)^{1 / 2}},
$$


where $A$ is the angle between the wind vector and the direction of motion of the beam induced by the telescope tracking.

The value of $t_{c}$ for each layer is measured from the autocorrelation of the WFS data. But there are three unknown quantities in Eq. 3: $h_{l}, v_{l}$, and $A$. To find all of them we make two measurements of $t_{c}$ with different values of $\rightarrow$, and also observe the change in the angle of each layer's motion through the beam. That is, the tomographic problem can be fully solved if the telescope tracks at a non-constant rate, provided that the layer ranges and velocities do not change significantly during the observations. This will naturally be the case for observations of most artificial satellites in non-geosynchronous orbits since the angular velocity of the telescope must vary to match the apparent track rate of the satellite across the sky.

The measurement geometry is illustrated in Figure 5. Solving the triangles in terms of the known and measured quantities is quite straightforward, and leads to a layer height

$$
h_{l}=\left[\frac{\phi}{D}+\frac{1}{R}\right]^{-1}
$$

where

$$
\phi=\left[\frac{\omega_{1}^{2}+\omega_{2}^{2}-2 \omega_{1} \omega_{2} \cos B}{t_{c 1}^{2}+t_{c 2}^{2}-2 t_{c 1} t_{c 2} \cos C}\right]^{1 / 2}
$$

Finding the actual layer velocities $\vec{v}_{l}$, if needed, is equally straightforward.

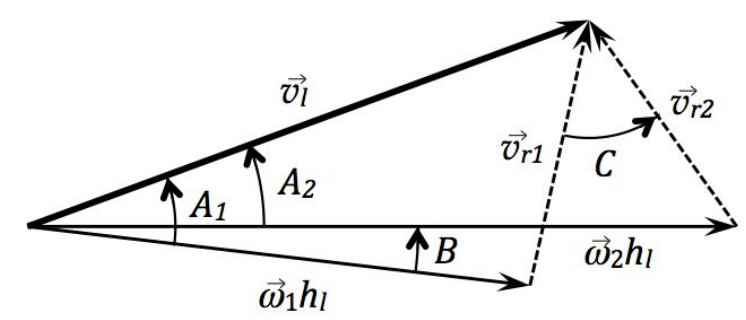

Figure 5. Vector geometry of the apparent velocities $\vec{v}_{r}$ of the guide star footprint through a layer at height $h_{l}$ moving with velocity $\vec{v}_{l}$ for two telescope track rates $\overrightarrow{1}_{1}$ and $\overrightarrow{ }_{2}$.

\section{RESULTS}

We have tested the estimation of layer heights using our four-layer simulation. We assumed telescope tracking rates $\overrightarrow{ }_{1}$ and $\overrightarrow{ }_{2}$ of $0.2 \%$ and $0.4 \%$, typical for low earth orbit satellites, with a $10^{\circ}$ difference in direction. The apparent layer velocities and crossing times were determined by the autocorrelation algorithm from our simulated Shack-Hartmann WFS data. The actual and measured values of the crossing times and orientation shift (angle $C$ in Figure 5) are given in Table 2. The recovered estimates of the layer heights and velocities are shown in Table 3. These are to be compared with the true values in Table 1.

In the case of a satellite tracked with a LGS our goal is to reduce the effects of focal anisoplanatism. This error term comprises three components: aberration at ranges beyond the beacon, unsensed aberration outside the cone defined by beacon light but encountered by light from the more distant object, and radial magnification of the sensed aberration as the beacon cone expands to fill the pupil. Our method does not address the first of these, which in any case is likely to be negligible, but the remaining two may be mitigated by respectively exploiting the FFM and our knowledge of the heights of significant layers.

Several methods have been suggested to take advantage of temporal coherence, ${ }^{13-15}$ including frozen flow characteristics, for improved wave-front control in AO systems. Here, the FFM allows us essentially to know the atmospheric aberration just outside the beacon light cone even when we cannot directly see it. Of course, we can only estimate aberration actually swept out by the beam footprint; some never will be as illustrated in Figure 6. Furthermore, in a closed-loop AO system, we can only know past history: the aberration that has already been sampled by the beacon and is now downstream. In that case we may benefit from pointing the laser slightly ahead of the object since in the case of satellites, a significant 
component of comes from the telescope tracking. In contrast, post-processing applications such as deconvolution from wave-front sensing can take advantage of both future and past history, as we have shown with data taken at the $3.6 \mathrm{~m}$ AEOS telescope. ${ }^{11}$

Table 2. Actual (measured) values of apparent layer wind vectors in simulation.

\begin{tabular}{cccc}
\hline Layer & $\boldsymbol{C}\left({ }^{\circ}\right)$ & $\boldsymbol{t}_{\boldsymbol{c} \mathbf{1}}(\mathbf{m s})$ & $\boldsymbol{t}_{\boldsymbol{c} \mathbf{1}(\mathbf{m s})}$ \\
\hline 1 & $0.0(0.1)$ & $2000(1620)$ & $2000(1818)$ \\
2 & $-5.3(-0.7)$ & $482(470)$ & $604(690)$ \\
3 & $-102.0(-104.2)$ & $451(481)$ & $265(276)$ \\
4 & $54.2(54.3)$ & $95(96)$ & $50(52)$ \\
\hline
\end{tabular}

Table 3. Results of the layer parameter estimation.

\begin{tabular}{crcc}
\hline Layer & Height $(\mathbf{m})$ & Direction & Speed $(\mathbf{m} / \mathbf{s})$ \\
\hline 1 & 74 & $1.2^{\circ}$ & 2.7 \\
2 & 750 & $2.1^{\circ}$ & 11.0 \\
3 & 4850 & $-9.5^{\circ}$ & 24.0 \\
4 & 14700 & $40.4^{\circ}$ & 49.4 \\
\hline
\end{tabular}

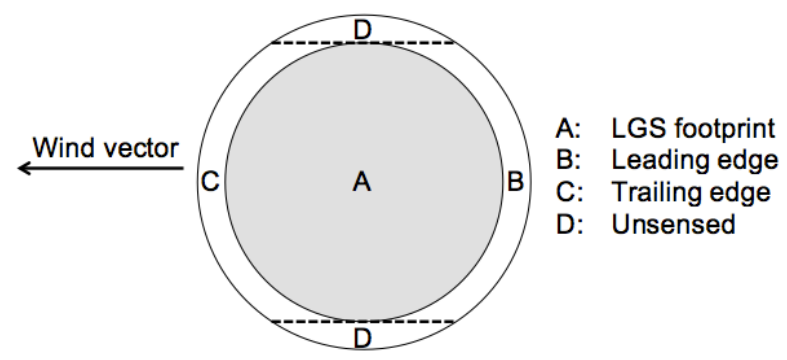

Figure 6. The beam footprint of the object light (outer circle) on a high-altitude layer is only partially sampled by the LGS (shaded region A) at any given moment. The regions labeled D are never sampled.

By placing the separated layers at their correct ranges, the beam compression ratio $h_{l} / R$ can be taken into account in deriving the best estimate of the aberration in the object light. The radial magnification arising from the cone effect can be removed. The quantitative reduction in anisoplanatic effects achievable in this way will depend on the prevailing turbulence profile and the extent to which multi-layer frozen-flow behavior can account for the short-term evolution of the wave front. For our model atmosphere in Table 1, the value of the focal anisoplanatism parameter $d_{0}$ is $1.9 \mathrm{~m} .{ }^{16} \mathrm{The}$ focal anisoplanatism error itself, given by $\lambda / 2 \pi\left(D / d_{0}\right)^{5 / 6}$, is $148 \mathrm{~nm}$ rms for our $4 \mathrm{~m}$ telescope, in satisfactory agreement with the value of $146 \mathrm{~nm}$ calculated directly from the 1000 composite phase screens of the simulation. We are able to reduce the error to $54 \mathrm{~nm}$ rms by taking advantage of the layer separation illustrated in Figure 4 and the layer characteristics calculated from tomography in Table 3 to estimate the object phase in regions A, B, and C of Figure 6 . We also explicitly estimate the unsensed portions of the object wave front (region D) via a set of 42 disk harmonic modes ${ }^{17}$ extending over the full object footprint, but fitted only to the estimated wave front within the LGS footprint.

This result is likely somewhat optimistic since the real atmosphere is not characterized by perfect frozen flow behavior, which we rely on to estimate the phase in regions B and C. For realistic wind speeds and a LGS at $90 \mathrm{~km}$, however, the crossing time for these regions is on the order of milliseconds, an order of magnitude shorter than $\tau_{\mathrm{FFM}}$. 


\section{DISCUSSION}

Focus anisoplanatism typically dominates the wave-front error budget for single-LGS AO systems. We expect that our method, where applicable, will reduce the effect of the error term to the point where that is no longer the case.

Even given the limitation that the FFM only allows phase estimation to be extended in the direction of each layer's wind vector, mitigation of angular anisoplanatism will be possible as well by placing the optical entrance pupil at some axial distance from the telescope, and slightly reducing its size. ${ }^{18}$ The smaller pupil allows less light into the image, but in return, the field of view over which the atmospheric aberration may be accurately sensed (and then corrected) may be substantially enlarged. The footprint of light from objects at infinity is arranged to stay within the sensed regions on all significant layers of aberration over a field of view roughly an order of magnitude larger than the isoplanatic angle.

\section{REFERENCES}

[1] T. Rimmele, S. Hegwer, K. Richards, F. Wöger, J. Marino, D. Schmidt, and T. Waldmann, "Solar multi-conjugate adaptive optics at the Dunn Solar Telescope," Advanced Maui Optical and Space Surveillance Technologies Conference p. E18 (2008).

[2] G. Moretto, M. Langlois, P. Goode, N. Gorceix, and S. Shumko, "Design for solar multi-conjugate adaptive optics at the new solar telescope/big bear solar observatory," Proceedings of the Third AO4ELT Conference p. 106 (2013).

[3] M. Lloyd-Hart, C. Baranec, N. M. Milton, M. Snyder, T. Stalcup, and J. R. P. Angel, "Experimental results of groundlayer and tomographic wavefront reconstruction from multiple laser guide stars," Opt. Exp. 14, 7541 (2006).

[4] J. Marino and F. Wöger, "Feasibility study of a layer-oriented wavefront sensor for solar telescope," Appl. Opt. 53, 685-693 (2014).

[5] M. Tallon and R. Foy, "Adaptive telescope with laser probe: isoplanatism and cone effect," Astron. Astrophys. 235, 549-557 (1990).

[6] F. J. Rigaut, B. L. Ellerbroek, and R. Flicker, "Principles, limitations, and performance of multiconjugate adaptive optics," Adaptive Optical Systems Technology (Proc. SPIE) 4007, 1022-1031 (2000).

[7] L. A. Poyneer, M. van Dam, and J.-P. Véran, "Experimental verification of the frozen flow atmospheric turbulence assumption with use of astronomical adaptive optics telemetry," J. Opt. Soc. Am. A 26, 833-846 (2009).

[8] E. Gendron and P. Léna, "Single layer atmospheric turbulence demonstrated by adaptive optics observations," Astrophys. Space Sci. 239, 221-228 (1996).

[9] E. Schöck and E. J. Spillar, "Method for a quantitative investigation of the frozen flow hypothesis," J. Opt. Soc. Am. A $17,1650-1658$ (2000).

[10] D. L. Fried, "Time-delay-induced mean-square error in adaptive optics," J. Opt. Soc. Am. A 7, 1224-1225 (1990).

[11] D. A. Hope, M. Hart, S. M. Jefferies, and J. G. Nagy, "Robust image restoration for ground-based space surveillance," Advanced Maui Optical and Space Surveillance Technologies Conference p. E51 (2012).

[12] S. M. Jefferies and M. Hart, "Deconvolution from wave-front sensing using the frozen flow hypothesis," Opt. Exp. 19, 1975-1984 (2011).

[13] K. Hinnen, M. Verhagen, and N. Doelman, "Exploiting the spatiotemporal correlation in adaptive optics using datadriven H2-optimal control," J. Opt. Soc. Am. A 24, 1714-1725 (2007).

[14] L. Poyneer and J.-P. Véran, "Predictive wavefront control for adaptive optics with arbitrary control loop delays," J. Opt. Soc. Am. A 25, 1486-1496 (2008).

[15] D. J. Goorskey, J. Schmidt, and M. R. Whiteley, "Efficacy of predictive wavefront control for compensating aerooptical aberrations," Opt. Eng. 52, 071418-1-071418-12 (2013).

[16] J. F. Belsher and D. L. Fried, "Expected antenna gain when correcting tilt-free wavefronts," Report TR-576, The Optical Sciences Company (1984). 
[17] N. M. Milton and M. Lloyd-Hart, "Disk harmonic functions for adaptive optics simulations," Adaptive Optics: Methods, Analysis and Applications (Proc. OSA symposium) (2005).

[18] M. Lloyd-Hart, "Extending the field of view of an adaptively corrected telescope by longitudinal pupil displacement," Opt. Lett. 27, 1469-1471 (2002).

Proc. of SPIE Vol. $9982998207-8$ 